\title{
Stable Splitting of Bivariate Splines Spaces by Bernstein-Bézier Methods
}

\author{
Oleg Davydov and Abid Saeed \\ Department of Mathematics and Statistics, \\ University of Strathclyde, Glasgow, United Kingdom \\ \{oleg.davydov, abid.saeed\}@strath.ac.uk \\ http://www.mathstat.strath.ac.uk/
}

\begin{abstract}
We develop stable splitting of the minimal determining sets for the spaces of bivariate $C^{1}$ splines on triangulations, including a modified Argyris space, Clough-Tocher, Powell-Sabin and quadrilateral macro-element spaces. This leads to the stable splitting of the corresponding bases as required in Böhmer's method for solving fully nonlinear elliptic PDEs on polygonal domains.
\end{abstract}

Keywords: Fully nonlinear PDE, Monge-Ampère equation, multivariate splines, Bernstein-Bézier techniques

\section{Introduction}

Numerical solution of fully nonlinear elliptic partial differential equations is a topic of intensive research and great practical interest, see [2,4]. Since no weak form formulation is available for the equations of this type in general, the standard Galerkin finite element method cannot be applied directly.

Recently, Böhmer [1,2] introduced a general approach that solves the Dirichlet problem for fully nonlinear elliptic equations numerically with the help of a sequence of linear elliptic equations used within an appropriate Newton scheme. These linear elliptic equations can be solved by the finite element method, but the discretisation has to be done by appropriate spaces of $C^{1}$ finite elements (splines) that admit a stable splitting into a subspace satisfying zero boundary conditions, and its complement. Such a stable splitting has been developed in [6] for a modified space of the Argyris finite element.

In this paper we systematically study the problem of stable splitting for the spaces of bivariate $C^{1}$ splines on triangulations of low degree using the BernsteinBézier methods. It turns out that stable splitting can be easily formulated as splitting of the minimal determining sets (MDS). We revisit the modified Argyris space studied in [6] by a different technique, and show that its modification is necessary at least if the convenient MDS splitting approach is used. We also show that Clough-Tocher, Powell-Sabin and quadrilateral macro-element spaces admit the stable splitting and therefore can also be used in the Böhmer's numerical method. 
The paper is organised as follows. Section 2 is devoted to an outline of Böhmer's method, whereas Section 3 introduces necessary definitions from the theory of Bernstein-Bézier methods [8], and defines the stable splitting of an MDS. In Section 4 we discuss the stable splitting for the Argyris space and its modification, and Section 5 is devoted to the $C^{1}$ macro-element spaces.

\section{Böhmer's Method for Fully Nonlinear Elliptic PDEs}

\subsection{Fully Nonlinear Elliptic Operators}

Let $\Omega$ be a bounded domain in $\mathbb{R}^{n}$ and let $G: H^{\gamma}(\Omega) \rightarrow L^{2}(\Omega), \gamma \geq 2$, be a second order differential operator of the form

$$
G(u)=\widetilde{G}\left(\cdot, u, \nabla u, \nabla^{2} u\right),
$$

where $\widetilde{G}$ is a real valued function defined on a domain $\widetilde{\Omega} \times \Gamma$ such that

$$
\bar{\Omega} \subset \widetilde{\Omega} \subset \mathbb{R}^{n} \text { and } \Gamma \subset \mathbb{R} \times \mathbb{R}^{n} \times \mathbb{R}^{n \times n},
$$

and $\nabla u, \nabla^{2} u$ denote the gradient and the Hessian of $u$, respectively. The points in $\widetilde{\Omega} \times \Gamma$ are denoted by $w=(x, z, p, r)$, with $x \in \widetilde{\Omega}, z \in \mathbb{R}, p=\left[p_{i}\right]_{i=1}^{n} \in \mathbb{R}^{n}$, $r=\left[r_{i j}\right]_{i, j=1}^{n} \in \mathbb{R}^{n \times n}$, to indicate the product structure of this set.

The operator $G$ is said to be elliptic in a subset $\widetilde{\Gamma} \subset \widetilde{\Omega} \times \Gamma$ if the matrix $\left[\frac{\partial \widetilde{G}}{\partial r_{i j}}(w)\right]_{i, j=1}^{n}$ is well defined and positive definite for all $w \in \widetilde{\Gamma}[2,7]$. If $\widetilde{G}$ is a linear function of $(z, p, r)$ for each fixed $x$, then $G$ is a linear differential operator. Under suitable restrictions on $\widetilde{G}$, classes of quasilinear and semilinear differential operators are obtained [2, p. 80], but in general $G$ may be fully nonlinear.

In the neighborhood of a fixed function $\hat{u} \in H^{\gamma}(\Omega)$ the linear elliptic operator $G^{\prime}(\hat{u})$ is defined by

$$
G^{\prime}(\hat{u}) u=\frac{\partial \widetilde{G}}{\partial z}(\hat{w}) u+\sum_{i=1}^{n} \frac{\partial \widetilde{G}}{\partial p_{i}}(\hat{w}) \partial^{i} u+\sum_{i, j=1}^{n} \frac{\partial \widetilde{G}}{\partial r_{i j}}(\hat{w}) \partial^{i} \partial^{j} u
$$

where $\hat{w}=\left(x, \hat{u}(x), \nabla \hat{u}(x), \nabla^{2} \hat{u}(x)\right)$ is a function of $x \in \Omega$, and $\partial^{i}$ denotes the partial derivative with respect to the $i$-th variable. If $G: H^{\gamma}(\Omega) \rightarrow L^{2}(\Omega)$ is Fréchet differentiable at $\hat{u}$, then $G^{\prime}(\hat{u}): H^{\gamma}(\Omega) \rightarrow L^{2}(\Omega)$ is its Fréchet derivative. If $G^{\prime}(\hat{u})$ depends continuously on $\hat{u}$ with respect to the linear operator norm, then $G$ is said to be continuously differentiable at $\hat{u}$.

Many nonlinear elliptic operators and corresponding equations $G(u)=0$ are important for applications, for example the Monge-Ampère equation for $\Omega \subset \mathbb{R}^{2}$, given by

$$
G_{\mathrm{MA}}(u):=\operatorname{det}\left(\nabla^{2} u\right)-f(x)=0, \quad f(x)>0 \text { for } x \in \Omega .
$$

The operator $G_{\mathrm{MA}}$ is fully nonlinear and $G_{\mathrm{MA}}(u) \in L^{2}(\Omega)$ if $u$ belongs to the Sobolev space $H^{5 / 2}(\Omega)$ and $f \in L^{2}(\Omega)$. Moreover, $G_{\mathrm{MA}}: H^{\gamma}(\Omega) \rightarrow L^{2}(\Omega)$ is continuously differentiable if $\gamma \geq 5 / 2$. 
We consider the Dirichlet problem for the operator $G$ : Find $u$ such that

$$
\begin{aligned}
G(u)=0, & x \in \Omega \\
u=\phi, & x \in \partial \Omega
\end{aligned}
$$

where $\phi$ is a continuous function defined on $\partial \Omega$. Under certain assumptions (including the exterior sphere condition for $\partial \Omega$ and sufficient smoothness of $\widetilde{G}$, satisfied in particular in the above mentioned examples if $f \in C^{2}(\Omega)$ ), this problem has a unique solution $u \in C^{2}(\Omega) \cap C(\bar{\Omega})$ [7, Theorem 17.17]. Note that the Monge-Ampère operator $G_{\mathrm{MA}}$ is elliptic in subsets $\widetilde{\Gamma}$ satisfying

$$
\widetilde{\Gamma} \subset \widetilde{\Omega} \times \mathbb{R} \times \mathbb{R}^{n} \times\left\{r \in \mathbb{R}^{n \times n}: r \text { is positive definite }\right\}
$$

Therefore there exists a unique convex solution of $G_{\mathrm{MA}}(u)=0$, whereas it is known that the Monge-Ampère equation has another, concave solution [3, Chapter 4].

\subsection{Spline Spaces and Stable Splitting}

As usual in the finite element method, the discretisation of the Dirichlet problem is done with the help of spaces of piecewise polynomial functions (splines). Let $\triangle$ be a triangulation of a polyhedral domain $\Omega \subset \mathbb{R}^{n}$, that is a partition of $\Omega$ into simplices such that the intersection of every pair of simplices is either empty or a common face. The space of multivariate splines of degree $d$ and smoothness $r$ is defined by

$$
S_{d}^{r}(\triangle)=\left\{s \in C^{r}(\Omega):\left.s\right|_{T} \in P_{d} \text { for all simplices } T \text { in } \triangle\right\},
$$

where $d>r \geq 0$ and $P_{d}$ is the space of polynomials of total degree $d$ in $n$ variables. Recall that the star of a vertex $v$ of $\triangle$, denoted by $\operatorname{star}(v)=\operatorname{star}^{1}(v)$, is the union of all triangles $T \in \triangle$ attached to $v$. We define $\operatorname{star}^{j}(v), j \geq 2$, inductively as the union of the stars of all vertices of $\triangle$ contained in $\operatorname{star}^{j-1}(v)$.

Let $\left\{\triangle^{h}\right\}_{h \in H}$ be a family of triangulations of $\Omega$, where $h$ is the maximum edge length in $\triangle^{h}$. The triangulations in the family are said to be quasi-uniform if there is an absolute constant $c>0$ such that $\rho_{T} \geq c h$ for all $T \in \triangle^{h}$, where $\rho_{T}$ denotes the radius of the inscribed sphere of the simplex $T$.

Let $S^{h} \subset S_{d}^{r}\left(\triangle^{h}\right)$ be a linear subspace with basis $s_{1}, \ldots, s_{N}$ and dual functionals $\lambda_{1}, \ldots, \lambda_{N}$ such that $\lambda_{i} s_{j}=\delta_{i j}$. This basis is stable and local if there are three constants $m \in \mathbb{N}$ and $C_{1}, C_{2}>0$ independent of $h$ such that (a) $\operatorname{supp} s_{k}$ is contained in $\operatorname{star}^{m}(v)$ for some vertex $v$ of $\triangle^{h}$, (b) $\left\|s_{k}\right\|_{L^{\infty}(\Omega)} \leq C_{1}$, $k=1, \ldots, N$, and (c) $\left|\lambda_{k} s\right| \leq C_{2}\|s\|_{L^{\infty}\left(\operatorname{supp} s_{k}\right)}, k=1, \ldots, N$, for all $s \in S^{h}$, see $[5,6]$ and $[2$, Section 4.2.6].

To handle the Dirichlet boundary conditions, the following subspace of $S^{h}$ is important:

$$
S_{0}^{h}:=\left\{s \in S^{h}:\left.s\right|_{\partial \Omega}=0\right\}
$$


Moreover, the method of solving (1)-(2) proposed in [1,2] requires a stable splitting of $S^{h}$ into a direct sum

$$
S^{h}=S_{0}^{h}+S_{b}^{h},
$$

such that a stable local basis $\left\{s_{1}, \ldots, s_{N}\right\}$ for $S^{h}$ can be split into two parts

$$
\left\{s_{1}, \ldots, s_{N}\right\}=\left\{s_{1}, \ldots, s_{N_{0}}\right\} \cup\left\{s_{N_{0}+1}, \ldots, s_{N}\right\},
$$

where $\left\{s_{1}, \ldots, s_{N_{0}}\right\}$ and $\left\{s_{N_{0}+1}, \ldots, s_{N}\right\}$ are bases for $S_{0}^{h}$ and $S_{b}^{h}$, respectively. Note that the space $S_{b}^{h}$ is not uniquely defined by the pair $S^{h}, S_{0}^{h}$. It was shown in [6] (see also [2, Section 4.2.6]) how the stable splitting can be achieved for a modified space of Argyris finite element.

\section{$2.3 \quad$ Böhmer's Method}

Let $u=\hat{u}$ be the solution of (1)-(2). According to [1], its approximation $\hat{u}^{h} \approx \hat{u}$ is sought as a solution of the following problem: Find $\hat{u}^{h} \in S^{h}$ such that

$$
\begin{aligned}
\left(G\left(\hat{u}^{h}\right), v^{h}\right)_{L^{2}(\Omega)} & =0 \quad \forall v^{h} \in S_{0}^{h}, \quad \text { and } \\
\left(\hat{u}^{h}, v_{b}^{h}\right)_{L^{2}(\partial \Omega)} & =\left(\phi, v_{b}^{h}\right)_{L^{2}(\partial \Omega)} \quad \forall v_{b}^{h} \in S_{b}^{h},
\end{aligned}
$$

where $(\cdot, \cdot)$ denotes the inner products in the respective Hilbert spaces. Since $S_{0}^{h}$ and $S_{b}^{h}$ are finite dimensional linear spaces, the problem (4)-(5) is equivalent to a system of algebraic equations with respect to the coefficients of $\hat{u}^{h}$ in a basis of $S^{h}$

Theorem 1 ([1, Theorem 8.7] and [2, Theorem 5.2]). Let $\Omega$ be a bounded convex polyhedral domain, and let $G: D(G) \rightarrow L^{2}(\Omega)$, with $D(G) \subset H^{2}(\Omega)$, satisfy Condition $H$ of [2, Section 5.2.3]. Assume that $G$ is continuously differentiable in the neighbourhood of an isolated solution $\hat{u}$ of $(1)-(2)$, such that $\hat{u} \in H^{\ell}(\Omega), \ell>2$, and $G^{\prime}(\hat{u}): D(G) \cap H_{0}^{1}(\Omega) \rightarrow L^{2}(\Omega)$ is boundedly invertible. Furthermore, assume that the spline spaces $S^{h} \subset S_{d}^{1}\left(\triangle^{h}\right), d \geq \ell-1$, on quasi-uniform triangulations $\triangle^{h}$ possess stable local bases and stable splitting $S^{h}=S_{0}^{h}+S_{b}^{h}$, and include polynomials of degree $\ell-1$. Then the problem (4)(5) has a unique solution $\hat{u}^{h} \in S^{h}$ as soon as the maximum edge length $h$ is sufficiently small. Moreover,

$$
\left\|\hat{u}-\hat{u}^{h}\right\|_{H^{2}(\Omega)} \leq C h^{\ell-2}\|\hat{u}\|_{H^{\ell}(\Omega)} .
$$

In particular, Condition H is satisfied by the Monge-Ampère operators on bounded convex polygonal domains in $\mathbb{R}^{2}$.

The nonlinear problem (4)-(5) can be solved iteratively by a Newton method [1], where the initial guess $u_{0}^{h} \in S^{h}$ satisfies the boundary condition

$$
\left(u_{0}^{h}, v_{b}^{h}\right)_{L^{2}(\partial \Omega)}=\left(\phi, v_{b}^{h}\right)_{L^{2}(\partial \Omega)} \quad \forall v_{b}^{h} \in S_{b}^{h},
$$


and the sequence of approximations $\left\{u_{k}^{h}\right\}_{k \in \mathbb{N}}$ of $\hat{u}^{h}$ is generated by

$$
u_{k+1}^{h}=u_{k}^{h}-w^{h}, \quad k=0,1, \ldots,
$$

with $w^{h} \in S_{0}^{h}$ being the solution of the linear elliptic problem:

$$
\text { Find } w^{h} \in S_{0}^{h} \text { such that }\left(G^{\prime}\left(u_{k}^{h}\right) w^{h}, v^{h}\right)_{L^{2}(\Omega)}=\left(G\left(u_{k}^{h}\right), v^{h}\right)_{L^{2}(\Omega)} \forall v^{h} \in S_{0}^{h} .
$$

Clearly, $w^{h}$ can be found by using the standard finite element method. Under some additional assumptions on $G$, it is proved in [1, Theorem 9.1] that $u_{i}^{h}$ converges to $\hat{u}$ quadratically. Note that in the case when $G(u)$ is only conditionally elliptic (e.g. elliptic only for a convex $u$ for Monge-Ampère equation) the ellipticity of the above linear problem is only guaranteed for $u_{k}^{h}$ sufficiently close to the exact solution $\hat{u}$.

\section{Bernstein-Bézier Techniques}

Certain spaces of bivariate $C^{1}$ splines with stable local bases and stable splitting required in Böhmer's method have been investigated by nodal techniques in [6]. However, Bernstein-Bézier methods are often preferable. Let us recall some related key concepts here, see [8] for more details.

From now on we only consider the bivariate case. In particular, $\Omega$ is a polygonal domain in $\mathbb{R}^{2}$ and $\triangle$ is a triangulation of $\Omega$.

Given $d \geq 1$, let $D_{d, \triangle}:=\bigcup_{T \in \triangle} D_{d, T}$ be the set of domain points, where

$$
D_{d, T}:=\left\{\xi_{i j k}=\frac{i v_{1}+j v_{2}+k v_{3}}{d}\right\}_{i+j+k=d}
$$

for each triangle $T:=\left\langle v_{1}, v_{2}, v_{3}\right\rangle$ in $\triangle$. Also note that every $v \in \mathbb{R}^{2}$ can be uniquely represented in the form

$$
v=\sum_{i=1}^{3} b_{i} v_{i}, \quad \sum_{i=1}^{3} b_{i}=1 .
$$

The triplet $\left(b_{1}, b_{2}, b_{3}\right)$ is called the barycentric coordinates of $v$ relative to the triangle $T:=\left\langle v_{1}, v_{2}, v_{3}\right\rangle$, and

$$
B_{i j k}^{d}(v):=\frac{d !}{i ! j ! k !} b_{1}^{i} b_{2}^{j} b_{3}^{k}, \quad i+j+k=d,
$$

are the Bernstein-Bézier basis polynomials of degree $d$ associated with triangle $T$. Every polynomial $p$ of total degree $d$ can be written uniquely as

$$
p=\sum_{i+j+k=d} c_{i j k} B_{i j k}^{d}
$$

where $c_{i j k}$ are the Bézier coefficients of $p$. For each $s \in S_{d}^{r}(\triangle)$ and $\xi=\xi_{i j k} \in$ $D_{d, \triangle}$ we denote by $c_{\xi}$ the coefficient $c_{i j k}$ of the restriction of $s$ to any triangle 
$T \in \triangle$ containing $\xi$. (Because of the continuity of $s$ the coefficient $c_{\xi}$ does not depend on the particular choice of such triangle.)

We now introduce two additional notations. We refer to the set

$$
R_{n}\left(v_{1}\right):=\left\{\xi_{i j k} \in D_{d, \triangle}: i=d-n\right\}, \quad 0 \leq n \leq d,
$$

of domain points as the ring of radius $n$ arround the vertex $v_{1}$ and refer to the set

$$
D_{n}\left(v_{1}\right):=\bigcup_{m=0}^{n} R_{m}\left(v_{1}\right)
$$

as the disk of radius $n$ arround the vertex $v_{1}$.

A key concept for dealing with spline spaces is that of a minimal determining set. Recall that the set $M \subset D_{d, \triangle}$ is a determining set for a linear space $S \subset$ $S_{d}^{r}(\triangle)$ if

$$
s \in S \text { and } c_{\xi}=0 \quad \forall \xi \in M \quad \Rightarrow \quad s=0,
$$

and $M$ is a minimal determining set (MDS) for the space $S$ if there is no smaller determining set. Then $\operatorname{dim} S$ equals the cardinality $\#\{M\}$ of $M$. Let

$$
\Gamma_{\eta}:=\left\{\xi \in M: c_{\eta} \text { depends on } c_{\xi}\right\},
$$

where we say that $c_{\eta}$ depends on $c_{\xi}, \xi \in M$, if the value of $c_{\eta}$ is changed when we change the value of $c_{\xi}$. A minimal determining set $M$ for a space $S$ is said to be local if there exists an absolute integer constant $\ell$ not depending on $\triangle$ such that

$$
\Gamma_{\eta} \subset \operatorname{star}^{\ell}\left(T_{\eta}\right) \quad \forall \eta \in D_{d, \triangle} \backslash M,
$$

where $T_{\eta}$ is a triangle containing $\eta$. And $M$ is called stable if there exists a constant $K$ which may depend only on $d, \ell$ and the smallest angle $\theta_{\triangle}$ in the triangulation $\triangle$ such that

$$
\left|c_{\eta}\right| \leq K \max _{\xi \in \Gamma_{\eta}}\left|c_{\xi}\right| \quad \forall \eta \in D_{d, \triangle} \backslash M .
$$

Given a stable local minimal determining set $M$ for $S \subset S_{d}^{r}(\triangle)$, a stable local basis $\left\{s_{\xi}\right\}_{\xi \in M}$ for $S$ can be defined by requiring that the Bézier coefficients $c_{\eta}$, $\eta \in M$, of $s_{\xi}$ satisfy $c_{\xi}=1$ and $c_{\eta}=0$ for all $\eta \in M \backslash\{\xi\}$, see [8, Section 5.8]. Clearly, a stable splitting of this basis is achieved by an appropriate splitting of the MDS, which leads to the following definition.

Definition 1. Assume that the space $S \subset S_{d}^{r}(\triangle)$ has a stable local MDS $M$ and let

$$
S_{0}:=\left\{s \in S:\left.s\right|_{\partial \Omega}=0\right\} .
$$

The MDS $M$ is said to admit a stable splitting if $M$ is the disjoint union of two subsets $M_{0}, M_{b} \subset M$ such that

$$
S_{0}=\left\{s \in S: c_{\xi}=0 \forall \xi \in M_{b}\right\}
$$

and $M_{0}$ and $M_{b}$ are stable local $M D S$ for the spaces $S_{0}$ and $S_{b}$, respectively, where

$$
S_{b}:=\left\{s \in S: c_{\xi}=0 \forall \xi \in M_{0}\right\}
$$


Note that if $M$ is a stable local MDS, and $M=M_{0} \cup M_{b}$ is a disjoint union, then it is a stable splitting as soon as (7) holds. Indeed, assume (7) is correct. If $s \in S_{0}$, then its coefficients related to $M_{b}$ are zero, and similarly if $s \in S_{b}$ then its coefficient related to $M_{0}$ are zero. Hence computing $s$ from coefficient corresponding to points in $M_{0}$ (respectively, $M_{b}$ ) is equivalent to computing from $M$, and so $M_{0}$ and $M_{b}$ are determining sets for $S_{0}$ and $S_{b}$, respectively. They are minimal determining sets because otherwise $M$ would not be minimal. Clearly, stability and locality properties of $M_{0}$ and $M_{b}$ are also inherited from $M$.

If $M$ admits a stable splitting, then $S=S_{0}+S_{b}$ and it is easy to see that

$$
\left\{s_{\xi}\right\}_{\xi \in M}=\left\{s_{\xi}\right\}_{\xi \in M_{0}} \cup\left\{s_{\xi}\right\}_{\xi \in M_{b}}
$$

is a stable splitting of the stable local basis $\left\{s_{\xi}\right\}_{\xi \in M}$.

\section{Stable Splitting for Argyris Finite Element}

Recall that the superspline subspaces $S_{d}^{r, \rho}(\triangle), r \leq \rho \leq d$, of $S_{d}^{r}(\triangle)$ are defined as

$$
S_{d}^{r, \rho}(\triangle)=\left\{s \in S_{d}^{r}(\triangle): s \in C^{\rho}(v) \forall v \in V\right\},
$$

where $V$ is the set of all vertices of $\triangle$.

Consider the Argyris finite element space obtained with $d=5, r=1$ and $\rho=2$ in (9). Now for each $v \in V$, let $T_{v}$ be any one of the triangles sharing the vertex $v$ and let $M_{v}:=D_{2}(v) \cap T_{v}$. For each edge $e$ of the triangulation $\triangle$, let $T_{e}:=\left\langle v_{1}, v_{2}, v_{3}\right\rangle$ be one of the triangles sharing the edge $e:=\left\langle v_{2}, v_{3}\right\rangle$ and let $M_{e}:=\left\{\xi_{122}^{T_{e}}\right\}$. Then from $[8$, Theorem 6.1] we have

Theorem 2. $\operatorname{dim} S_{5}^{1,2}(\triangle)=6 \#\{V\}+\#\{E\}$ and

$$
M=\bigcup_{v \in V} M_{v} \cup \bigcup_{e \in E} M_{e}
$$

is a stable local minimal determining set for $S_{5}^{1,2}(\triangle)$.

An example is given in Figure 1 (left).

\subsection{Modified Argyris Space}

We now modify the Argyris space to achieve the stable splitting. This construction is discussed in term of nodal basis functions in [6]. We will explain in Section 4.3 why this modification is required. Let us denote the modified Argyris space by $\tilde{S}$, where

$$
\tilde{S}:=\left\{s \in S_{5}^{1}(\triangle): s \in C^{2}(v), \text { for all interior vertices } v \text { of } \triangle\right\} .
$$

Let us now differentiate between boundary vertices and interior vertices by using $V_{I}$ and $V_{B}$ for the sets of interior and boundary vertices respectively. And let $E_{I}$ and $E_{B}$ denote interior and boundary edges respectively, such that

$$
V=V_{I} \cup V_{B}, \quad E=E_{I} \cup E_{B} .
$$


We describe a minimal determining set $\tilde{M}$ for this modified space $\tilde{S}$. Since we have modified the space only at the boundary vertices, so the points in $M$ related to interior vertices and related to all edges, will belong to $\tilde{M}$. That is,

$$
\left(\bigcup_{v \in V_{I}} M_{v} \cup \bigcup_{e \in E} M_{e}\right) \subset \tilde{M} .
$$

However, we will have to modify the sets corresponding to the boundary vertices $v \in V_{B}$. First of all, we require that each $T_{v}, v \in V_{B}$, is a triangle sharing an edge with the boundary of $\Omega$ (we call it a boundary triangle). Furthermore, we add some more points to $M_{v}, v \in V_{B}$, as follows. Let us denote all edges of $\triangle$ emanating from a vertex $v \in V_{B}$, in counterclockwise order, by

$$
E_{v}=\left\{e_{1}, e_{2}, \cdots, e_{n}\right\} .
$$

Then clearly $e_{1}, e_{n} \in E_{B}$, and the triangle $T_{v}$ is formed by either $e_{1}, e_{2}$ or $e_{n-1}, e_{n}$. For each $e_{i}$, let $\xi_{i}$ be the (unique) domain point in $R_{2}(v) \cap e_{i}, i=$ $1, \ldots, n$. We set

$$
\tilde{M}_{v}:=M_{v} \cup\left\{\xi_{1}, \xi_{2}, \cdots, \xi_{n}\right\} .
$$

Theorem 3. $\operatorname{dim} \tilde{S}=6 \#\left\{V_{I}\right\}+\#\{E\}+\sum_{v \in V_{B}}\left(4+\# E_{v}\right)$ and

$$
\tilde{M}:=\bigcup_{v \in V_{I}} M_{v} \cup \bigcup_{e \in E} M_{e} \cup \bigcup_{v \in V_{B}} \tilde{M}_{v} .
$$

is stable local MDS for modified Argyris space $\tilde{S}$.

Proof. We set the coefficients $\left\{c_{\xi}\right\}_{\xi \in \tilde{M}}$ for any spline $s \in \tilde{S}$ to arbitrary values and show that all other coefficients, i.e. $\left\{c_{\xi}\right\}_{\xi \in D_{5, \Delta} \backslash \tilde{M}}$, of $s$ can be determined consistently.

Now first note that for each $v \in V_{I}$ and for each $e \in E$ the points in $M_{v}$ and $M_{e}$ are the same as for Argyris space. So we only need to prove that for each $v \in V_{B}$ the set $\tilde{M}_{v}$ is an MDS on $D_{2}(v)$. To this end, for each $v \in V_{B}$, we set the coefficients of $s$ corresponding to points in $\tilde{M}_{v}$ and see that, in view of $C^{1}$ smoothness conditions, all coefficients corresponding to domain points in $D_{2}(v)$ can be determined consistently. Thus by $[8$, Theorem 5.15] $\tilde{M}$ is minimal determining set for the space $\tilde{S}$. Observe that $\tilde{M}$ is a stable MDS. Indeed, for each $v \in V_{I}$ and all edges $e \in E$ the stability follows from [8, Lemma 2.29]. And for each $v \in V_{B}$ the set $\tilde{M}_{v}$ is a stable MDS for $S_{5}^{1}$ on $D_{2}(v)$ by [8, Theorem 11.7]. Standard arguments show that $\tilde{M}$ is local.

The minimal determining sets for Argyris space and for modified Argyris space over a small triangulation with nine triangles are illustrated in Figure 1.

\subsection{Stable Splitting}

Now we show how to determine a stable splitting $\tilde{M}=\tilde{M}_{0} \cup \tilde{M}_{b}$ of the MDS $\tilde{M}$ for modified Argyris space $\tilde{S}$. 

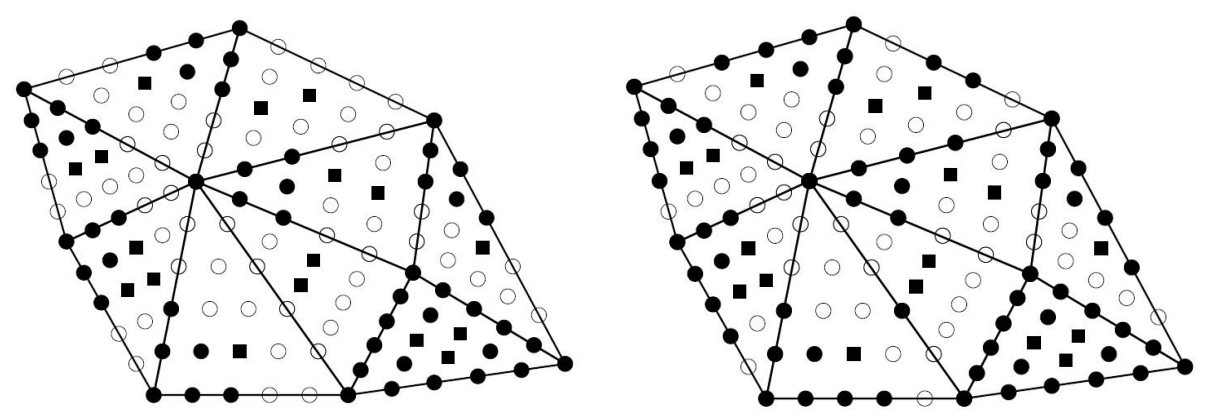

Fig. 1. Minimal determining sets for the Argyris space (left) and for the modified modified Argyris space (right). The points in the sets $M_{v}, \tilde{M}_{v}$ are marked by black dots, and those in $M_{e}$ by black squares.

It is already understood that all those points of $\tilde{M}$ which are on the boundary will be in $\tilde{M}_{b}$ and those points lying in $M_{v}, v \in V_{I}$, and $M_{e}$ along with the points in $R_{2}(v), v \in V_{B}$, but not on either $e_{1}$ or $e_{n}$, will be in $\tilde{M}_{0}$. Consider, for each $v \in V_{B}$, the remaining point which lies in $R_{1}(v), v \in V_{B}$, but not on the boundary edges. We denote this point by $\xi_{v}$. Whether $\xi_{v}$ belongs to $\tilde{M}_{0}$ or $\tilde{M}_{b}=\tilde{M} \backslash \tilde{M}_{0}$ depends on the geometry of the boundary edges $e_{1}$ and $e_{n}$, as follows.

- If $e_{1}$ and $e_{n}$ are non-collinear, then $\xi_{\sim} \in \tilde{M}_{b}$.

- If $e_{1}$ and $e_{n}$ are collinear, then $\xi_{v} \in \tilde{M}_{0}$.

Indeed, in the non-collinear case the coefficient corresponding to $\xi_{v}$ is zero for all $s \in \tilde{S}_{0}$, wheras in the collinear case it can be chosen freely. The Figures 2 and 3 show points in $\tilde{M}_{0}$ and $\tilde{M}_{b}$ for the boundary vertex with collinear and non-collinear edges respectively.
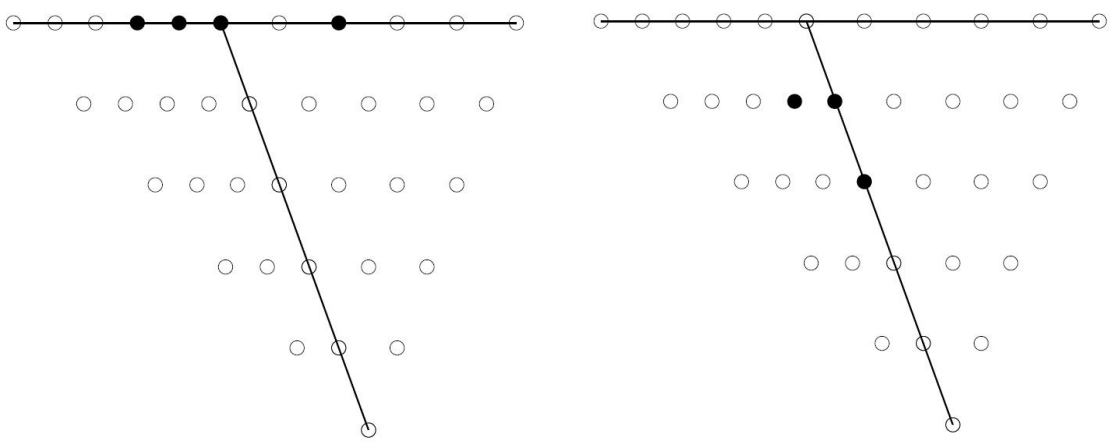

Fig. 2. Splitting of points in $\tilde{M}_{v}, v \in V_{B}$ for modified Argyris space with collinear boundary edges. Left: $\tilde{M}_{v} \cap \tilde{M}_{b}$, right: $\tilde{M}_{v} \cap \tilde{M}_{0}$. 

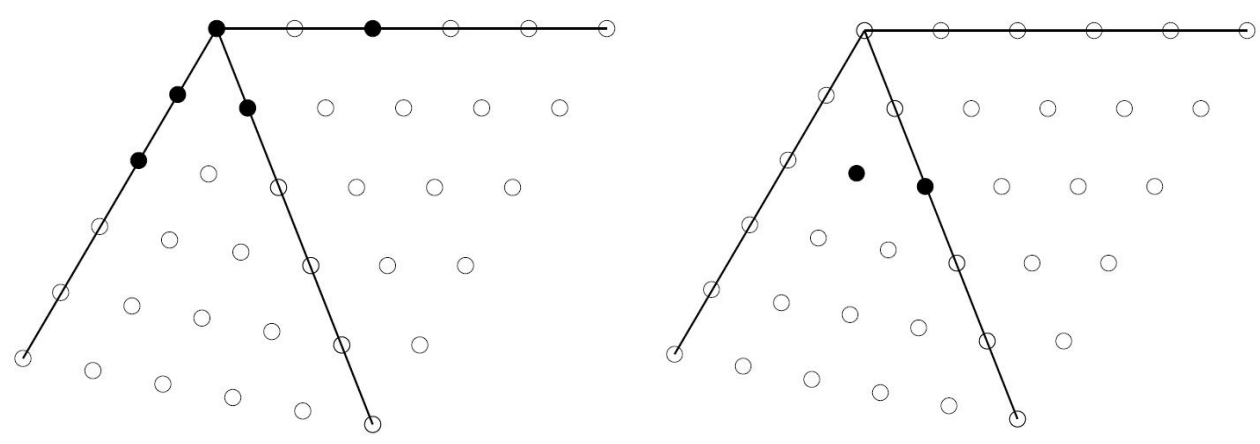

Fig. 3. Splitting of points in $\tilde{M}_{v}, v \in V_{\tilde{N}_{\tilde{N}}}$ for modified Argyris space with noncollinear boundary edges. Left: $\tilde{M}_{v} \cap \tilde{M}_{b}$, right: $\tilde{M}_{v} \cap \tilde{M}_{0}$.

Theorem 4. $\tilde{M}=\tilde{M}_{0} \cup \tilde{M}_{b}$ is stable splitting of MDS $\tilde{M}$.

Proof. If $s \in \tilde{S}_{0}$, then all its Bézier coefficient on the boundary are zero since $\left.s\right|_{\partial \Omega}=0$. For those $v \in V_{B}$ where the boundary edges are non-collinear, the $C^{1}$ smoothness implies that the gradient at $v$ is also zero, and hence the coefficient of $s$ at $\xi_{v}$ is also zero. This shows that $\tilde{S}_{0} \subset\left\{s \in \tilde{S}: c_{\xi}=0 \forall \xi \in \tilde{M}_{b}\right\}$. Conversely, assume $s \in \tilde{S}$ and $c_{\xi}=0$ for all $\xi \in \tilde{M}_{b}$. Let $v \in V_{B}$ and $E_{v}=\left\{e_{1}, e_{2}, \cdots, e_{n}\right\}$ as before. Without loss of generality assume that $D_{2}(v) \cap e_{1} \subset \tilde{M}_{v}$ and $R_{2}(v) \cap e_{n} \subset$ $\tilde{M}_{v}$. Therefore $c_{\xi}=0$ at all these points. However, due to the $C^{1}$ smoothness $c_{\xi}=0$ also for the domain point in $R_{1}(v) \cap e_{n}$, both in the collinear and noncollinear case. This shows that $c_{\xi}=0$ for all domain points on the boundary of $\Omega$ and hence $\left.s\right|_{\partial \Omega}=0$. Thus, $\tilde{S}_{0}=\left\{s \in \tilde{S}: c_{\xi}=0 \forall \xi \in \tilde{M}_{b}\right\}$, which completes the proof, see the discussion following Definition 1.

\subsection{Why Modification in Argyris Space is Required}

We now prove that modification is needed in Argyris space at the boundary vertices to achieve a stable splitting.

We first consider the Argyris space $S_{5}^{1,2}(\triangle)$ with $M$ in Theorem 2 being its MDS, and show that no splitting $M=M_{0} \cup M_{b}$ is possible in this case if there is a boundary vertex $v$ with two triangles attached, and the boundary edges are non-collinear. On contrary, assume that such a splitting has been found. Let $T:=\left\langle v_{1}, v_{2}, v_{3}\right\rangle$ and $\tilde{T}:=\left\langle v_{4}, v_{3}, v_{2}\right\rangle$ be two triangles in $\triangle$ with $v_{3}$ as boundary vertex and assume that the edges $\left\langle v_{3}, v_{4}\right\rangle$ and $\left\langle v_{3}, v_{1}\right\rangle$ are boundary edges. Consider the set

$$
M_{v_{3}}:=D_{2}\left(v_{3}\right) \cap T=\left\{\xi_{005}, \xi_{014}, \xi_{023}, \xi_{104}, \xi_{113}, \xi_{203}\right\} \subset M,
$$

see the Figure 4, and let

$$
\left.s\right|_{T}=\sum_{i+j+k=5} c_{i j k} B_{i j k}^{5},\left.\quad s\right|_{\tilde{T}}=\sum_{i+j+k=5} \tilde{c}_{i j k} \tilde{B}_{i j k}^{5},
$$


where $B_{i j k}^{5}$ and $\tilde{B}_{i j k}^{5}$ are Bernstein basis polynomials associated with $T$ and $\tilde{T}$ respectively. In the case that the edges $\left\langle v_{3}, v_{4}\right\rangle$ and $\left\langle v_{3}, v_{1}\right\rangle$ are non-collinear, the points $\left\{\xi_{005}, \xi_{014}, \xi_{104}, \xi_{203}\right\}$ must be in $M_{b}$, because $s \in S$ has zero coefficients at these points. We show that $\left\{\xi_{113}, \xi_{023}\right\} \not \subset M_{0}$. Let $\left(b_{1}, b_{2}, b_{3}\right)$ be barycentric coordinates of $v_{4}$ relative to $T$. Then by a $C^{2}$ smoothness condition, see $[8$, Theorem 2.28], across the edge $e:=\left\langle v_{3}, v_{2}\right\rangle$ we can write

$$
\tilde{c}_{230}=b_{1}^{2} c_{203}+2 b_{1} b_{2} c_{113}+2 b_{2} b_{3} c_{014}+b_{2}^{2} c_{023}+2 b_{1} b_{3} c_{104}+b_{3}^{2} c_{005}
$$

and because $\tilde{c}_{230}=c_{203}=c_{014}=c_{104}=c_{005}=0$,

$$
0=2 b_{1} c_{113}+b_{2} c_{023}
$$

which shows that $c_{113}$ and $c_{023}$ are linearly dependent so that $\xi_{113}, \xi_{023}$ cannot be both in $M_{0}$. Moreover, we cannot shift one of these points to $M_{b}$ because there is a spline $s \in S_{0}$ such that

$$
c_{113}, c_{023} \neq 0 \text {, }
$$

e.g. $s$ with $c_{113}=b_{2}$ and $c_{023}=-2 b_{1}$. Note that $b_{2} \neq 0$ if the boundary edges are non-collinear.

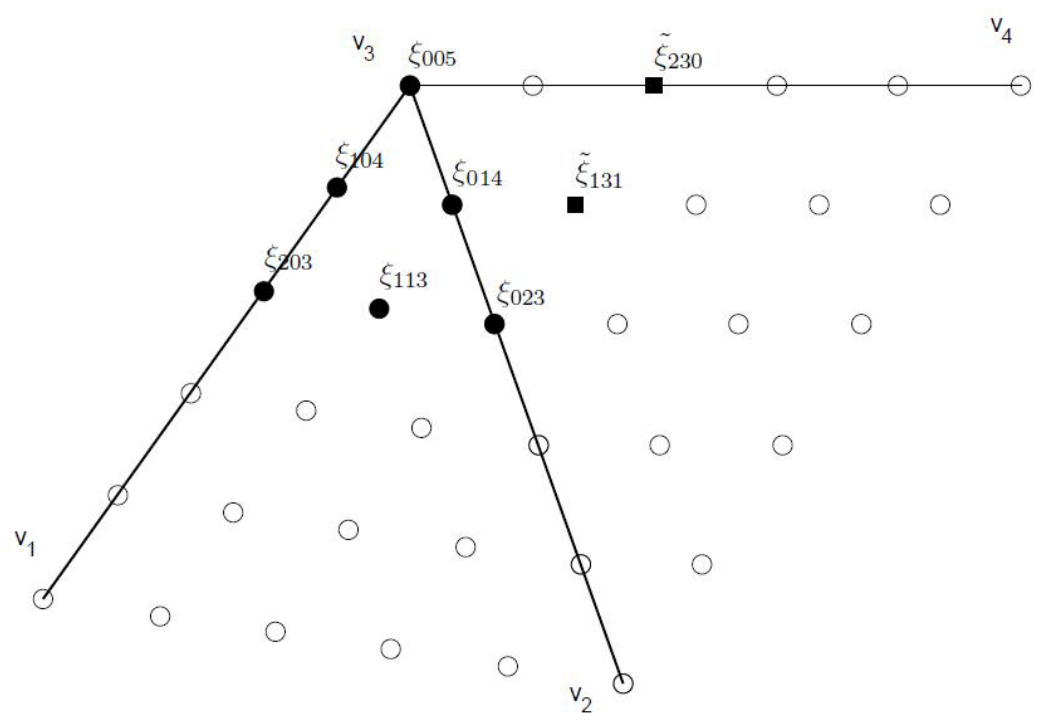

Fig. 4. The black dots are MDS points in $M_{v_{3}}, v_{3} \in V_{B}$, for Argyris space. The two domain points marked by black squares are involved in the smoothness conditions discussed in the proof of Theorem 5 .

Moreover, we prove that no other MDS admits a stable splitting, either. 
Theorem 5. No MDS for the Argyris space can be stably split on arbitrary triangulations.

Proof. Assume that the triangulation $\triangle$ is such that there is a boundary vertex $v$ with two triangles $T$ and $\tilde{T}$ attached, and the boundary edges are non-collinear at $v$, as in the above proof. Let $M$ be some MDS for Argyris space.

From the dimension argument we know that there must be exactly six points in $M \cap D_{2}(v)$. For the non-collinear boundary edges, no points on boundary edges or in $R_{1}(v)$ can be in $M_{0}$ because, all the corresponding coefficients of splines in $S_{0}$ are zero. So the only candidates for $M_{0}$ are the points in $R_{2}(v)$ not on boundary edges. Now we discuss the relation between the coefficients $\tilde{c}_{131}, c_{113}, c_{023}$ of $s \in S_{0}$ at these points. By using $C^{1}$ and $C^{2}$ condition across the common edge of $T$ and $\tilde{T}$ we get

$$
\begin{aligned}
\tilde{c}_{131} & =b_{1} c_{113}+b_{2} c_{023} \\
0 & =2 b_{1} c_{113}+b_{2} c_{023}
\end{aligned}
$$

By subtracting these equations we can write

$$
\tilde{c}_{131}=-b_{1} c_{113}
$$

Hence the three coefficients cannot be set arbitrarily. Only one of them can be chosen freely, which cannot be either $\tilde{c}_{131}$ or $c_{113}$. Indeed, let us choose e.g. $c_{113}$ arbitrarily, then from the above equations we obtain

$$
c_{023}=\frac{-2 b_{1} c_{113}}{b_{2}}
$$

and hence $c_{023} \rightarrow \infty$ for $b_{2} \rightarrow 0$ as the boundary edges get collinear. This would be unstable as the minimum angles in $T, \tilde{T}$ do not degenerate.

Thus $\xi_{023}$ is the only point to be in $M_{0}$. It is easy to see that $M_{b}$ must contain $\xi_{203}, \tilde{\xi}_{230}$ and three points in $D_{1}(v)$. Consider the basis spline $s$ in $S_{b}$ corresponding to $\tilde{\xi}_{230}$. Then its coefficient satisfy

$$
\tilde{c}_{230}=1, \quad c_{203}=c_{023}=0, \quad c_{\xi}=0, \quad \xi \in D_{1}(v)
$$

Now again using $C^{1}$ and $C^{2}$ conditions we find

$$
\tilde{c}_{230}=2 b_{1} b_{2} c_{113} \text { or } c_{113}=\frac{1}{2 b_{1} b_{2}},
$$

which is unbounded for $b_{2} \rightarrow 0$ as the boundary gets flat.

Remark 1. If a boundary vertex $v$ has exactly two triangles attached and the boundary edges are not collinear at $v$, then stable splitting of an MDS is impossible for any spline space $S$ where each spline is $C^{2}$ continuous at $v$. Indeed, this follows by the arguments in the proof of Theorem 5 . In fact, it is easy to see that the set $D_{2}(v) \cap T$ as MDS for $S$ on $D_{2}(v)$ cannot be split stably for a boundary vertex with any number of triangles attached. 


\section{$5 \quad C^{1}$ Macro-element Spaces}

Now we discuss the possibility of stable splitting of minimal determining sets of some of the $C^{1}$ macro-element spaces.

\subsection{Stable Splitting of Clough-Tocher Macro-element Space}

Given a triangulation $\triangle$ of a domain $\Omega$, let $\triangle_{C T}$ be corresponding CloughTocher refinement of $\triangle$, where each triangle is split into three subtriangles, see Figure 5.

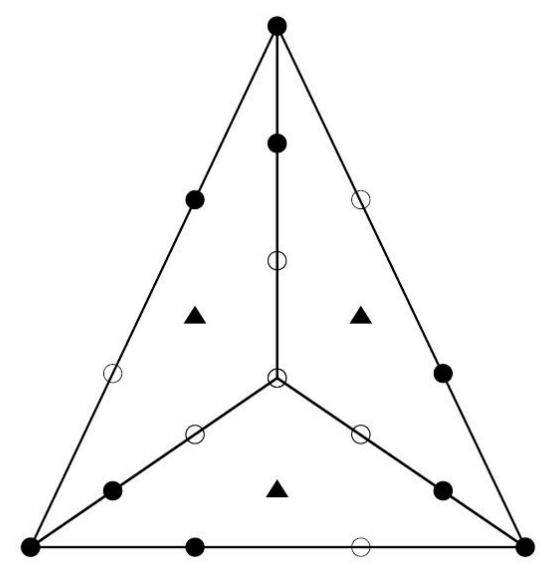

Fig. 5. A typical Clough-Tocher refinement of one triangle with points in $M_{v}$ marked as black dots and points in $M_{e}$ marked as black triangles.

Consider the stable local MDS $M$ given in [8, Theorem 6.5] for $C^{1}$ CloughTocher Macro-element space $S_{3}^{1}\left(\triangle_{C T}\right)$ as

$$
M=\bigcup_{v \in V} M_{v} \cup \bigcup_{e \in E} M_{e}
$$

where $M_{v}:=D_{1}(v) \cap T_{v}$ and $M_{e}:=\left\{\xi_{111}^{T_{e}}\right\}$, and $T_{v}$ and $T_{e}$ are triangles in $\triangle_{C T}$. Denote by $V$ and $E$ the sets of vertices and edges in $\triangle$, respectively. Let

$$
S_{0}:=\left\{s \in S_{3}^{1}\left(\triangle_{C T}\right):\left.s\right|_{\partial \Omega}=0\right\} .
$$

Let $V_{I}$ and $V_{B}$ be the sets of interior and boundary vertices of $\triangle$, respectively. We assume that $T_{v}$ is a boundary triangle for each $M_{v}, v \in V_{B}$. Then stable splitting for $M$ is possible as follows. Clearly,

$$
\left(\bigcup_{v \in V_{I}} M_{v} \cup \bigcup_{e \in E} M_{e}\right) \subset M_{0} .
$$


However, $M_{0}$ may contain some more points from $M_{v}, v \in V_{B}$. Note that, for boundary vertices $v$, two points in $M_{v}$ are always on the boundary and one is not. These two boundary points are in $M_{b}$ but the point in $M_{v}$, which is not on the boundary, belongs to either $M_{0}$ or $M_{b}$ depending on the geometry of boundary edges attached to $v$ in the same way as the point $\xi_{v}$ in Section 4.2. This point will be in $M_{0}$ for those boundary vertices where boundary edges are collinear. Otherwise it will be in $M_{b}$. Stability and locality follows as $M$ is a stable local MDS for $S_{3}^{1}\left(\triangle_{C T}\right)$.

\subsection{Powell-Sabin Macro-element Space}

Now let for a given triangulation $\triangle$ of a domain $\Omega, \triangle_{P S}$ be the corresponding Powell-Sabin refinement [8, Definition 4.18], see the Figure 6. For each $v \in V$, let $T_{v}$ be some triangle of $\triangle_{P S}$ attached to $v$, and $M_{v}:=D_{1}(v) \cap T_{v}$. Then

$$
M=\bigcup_{v \in V} M_{v}
$$

is a stable local minimal determining set for Powell-Sabin space $S_{2}^{1}\left(\triangle_{P S}\right)[8$, Theorem 6.9]. Now similarly if

$$
S_{0}:=\left\{s \in S_{2}^{1}\left(\triangle_{P S}\right):\left.s\right|_{\partial \Omega}=0\right\}
$$

and if we take $T_{v}$ to be a boundary triangle for $M_{v}, v \in V_{B}$, then $M$ given in (15) for $S_{2}^{1}\left(\triangle_{P S}\right)$ can be split stably in the same way as discussed above for the Clough-Tocher macro-element space.

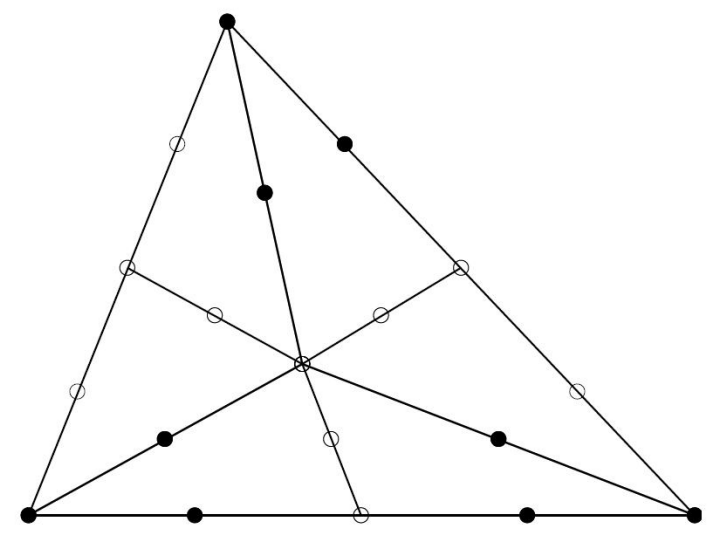

Fig. 6. Powell-Sabin refinement of one triangle with points in $M_{v}$ marked as black dots. 


\subsection{Powell-Sabin-12 Macro-element Space}

Let $\triangle_{P S 12}$ be the Powell-Sabin-12 refinement [8, Definition 4.21] of a given triangulation $\triangle$ of a domain $\Omega$, see Figure 7 . For each $e$ of $\triangle$, let $u_{e}$ be the midpoint of $e$ and let $v_{T}$ be the incenter of a triangle $T$ in $\triangle$ attached to $e$. Let $\xi_{e}:=\frac{v_{T}+u_{e}}{2}$ and $M_{e}:=\left\{\xi_{e}\right\}$. For each vertex $v \in V$, let $T_{v}$ be a triangle of $\triangle_{P S 12}$ attached to $v$, and let $M_{v}:=D_{1}(v) \cap T_{v}$. Then the set

$$
M=\bigcup_{v \in V} M_{v} \cup \bigcup_{e \in E} M_{e}
$$

is a stable local MDS for the space $S_{2}^{1}\left(\triangle_{P S 12}\right)$ [8, Theorem 6.13]. Now let

$$
S_{0}:=\left\{s \in S_{2}^{1}\left(\triangle_{P S 12}\right):\left.s\right|_{\partial \Omega}=0\right\} .
$$

Again, assuming that $T_{v}$ is a boundary triangle of $\triangle_{P S 12}$ for any bondary vertex $v$, we can split $M$ into $M_{0}$ and $M_{b}$ by the same method as for the Clough-Tocher elements. Then $M=M_{0} \cup M_{b}$ is a stable splitting for $S_{2}^{1}\left(\triangle_{P S 12}\right)$.

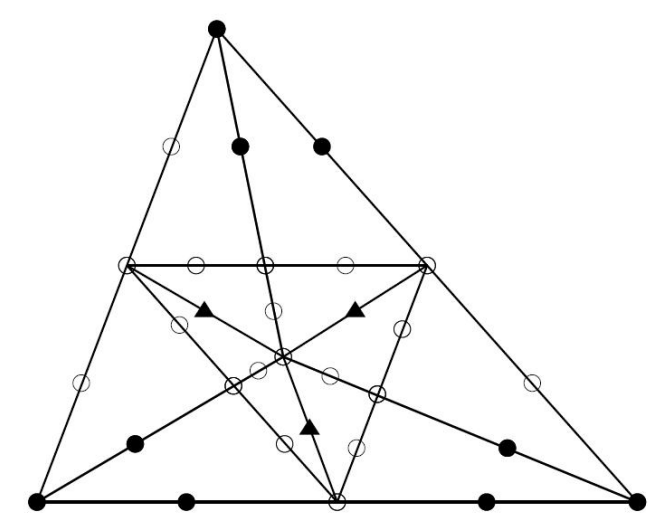

Fig. 7. A Powell-Sabin-12 refinement of one triangle with points in $M_{v}$ marked as black dots and points in $M_{e}$ marked as black triangles.

\subsection{Quadrilateral Macro-element Space}

Let $\diamond$ be a strictly convex quadrangulation of a polygonal domain $\Omega$ and let $\triangle_{Q}$ be triangulation obtained by drawing in the diagonals of each quadriletral of $\diamond$. Let $V$ and $E$ be the sets of vertices and edges of $\nabla$. Here we will discuss the cubic spline space $S_{3}^{1}\left(\triangle_{Q}\right)$. Again let $M_{v}:=D_{1}(v) \cap T_{v}$, for each $v \in V$, where $T_{v}$ is a triangle in $\triangle_{Q}$ attached to $v$, and $T_{v}$ is a boundary triangle in case of a 
boundary vertex $v$. For each $e \in E$, let $T_{e}$ be some triangle in $\triangle_{Q}$ containing $e$ and let $M_{e}:=\left\{\xi_{111}^{T_{e}}\right\}$. Then

$$
M=\bigcup_{v \in V} M_{v} \cup \bigcup_{e \in E} M_{e}
$$

is a stable local MDS for the space $S_{3}^{1}\left(\triangle_{Q}\right)$ [8, Theorem 6.17]. Again the stable splitting of $M$ for $S_{3}^{1}\left(\triangle_{Q}\right)$ is possible by the argument discussed above for other $C^{1}$ macro-elements.

Note that in $[8$, Section 6.5$]$ the above triangle $T_{v}$ is chosen such that it has the largest shape ratio $\operatorname{diam}(T) / \rho(T)$ among all triangles attached to $v$. This allows stable MDS even in the presence of small angles in $\triangle_{Q}$ if the smallest angle in $\diamond$ is separated from zero. However, this choice of $T_{v}$ might be unsuitable for stable splitting if $v$ is a boundary vertex because we need $T_{v}$ to be a boundary triangle whereas the shape ratio might be larger for some interior triangle attached to $v$. Therefore, our construction of stable splitting is valid only if $\triangle_{Q}$ satisfies the minimum angle condition.

\section{References}

1. K. Böhmer, On finite element methods for fully nonlinear elliptic equations of second order, SIAM J. Numer. Anal., 46 (3) (2008), 1212-1249.

2. K. Böhmer, Numerical Methods for Nonlinear Elliptic Differential Equations: A Synopsis, Oxford University Press, Oxford, 2010.

3. R. Courant and D. Hilbert, Methods of Mathematical Physics, vo.l II, Wiley Interscience, 1989.

4. E.J. Dean, R. Glowinski, Numerical methods for fully nonlinear elliptic equations of the Monge-Ampère type, Computer Methods in Applied Mechanics and Engineering, 195 (2006), 1344-1386

5. O. Davydov, Stable local bases for multivariate spline spaces, J. Approx. Theory, 111 (2001), 267-297.

6. O. Davydov, Smooth finite elements and stable splitting, Berichte "Reihe Mathematik" der Philipps-Universität Marburg, 2007-4 (2007). An adapted version has appeared as [2, Section 4.2.6].

7. D. Gilbarg and N. S. Trudinger, Elliptic partial differential equations of second order, Springer-Verlag, Berlin, (2001).

8. M. J. Lai and L. L. Schumaker, Spline Functions on Triangulations, Cambridge University Press, (2007). 